patient slept, when they would migrate to the superficial vessels where they were easily found.

In this condition the lymph-vessels are greatly dilated because of the blocking or choking of these channels, forming a varix, usually around the base or pedicle, together with large blood-spaces or sinuses, affording an excess amount of pabulum to the structures supplied, which accounts for the rapid growth of connective tissue. Accompanying this lymphedema, wherever there is a break in the continuity of the surface, there is usually a profuse lymphorrhea.

There is a condition caused by syphilis known as syphilis infiltrous in which the pathologic findings are identical with the foregoing, but in which the filaria is absent, and after numerous consultations I concluded this to be the correct diagnosis in this case.

Treatment.-Having arrived at this diagnosis, I determined to remove these growths and this was done by making an incision through the skin on the outer side of each growth the shape of the letter $Y$ inverted so that the flap to be left had the appearance of an inverted $\mathrm{V}$. This was done to allow for the retraction incident to the weight. Large venous blood-spaces were encountered with every stroke of the knife. They were easily controlled with clamps and many did not require ligatures. Care was taken not to injure the vaginal pouch, which I found pulled down and almost obliterated, but which retracted to a certain extent as soon as the growths were removed. A very satisfactory approximation of the skin-flaps was secured, rubber drainage-tubes were left in situ, and the usual dressings applied. In order that we might watch the immediate result of the operation, salvarsan was not given for one week, at the end of which time there was a general lymphedema and a profuse lymphorrhea with every indication of a rapid return of the condition. One week after the administration of salvarsan a marked improvement was noted, and in three weeks after the operation, a second dose was given. This was followed by a prompt disappearance of the edema, and the patient was soon able to be about her duties. The combined weight of the growths was 12 pounds. The patient passed from my observation in May when she left the city jail to serve a sentence in the state penitentiary.

Recently I was given an opportunity to secure a photograph which shows the patient to be in a very satisfactory condition. The labia are absent and only a small portion of the clitoris is left. The vagina is normal, and the patient has no leukorrhea or discomfort of any kind. Her general condition is excellent.

701 Grace Street West.

Traumatic Neuroses.-A. Ciampolini, the medical inspector for the state railroad system of Italy, has recertly published in Ramazzini, 1914, viii, 1-108, an analytic study of 160 out of a total of 200 cases of neurosis following railroad accidents. The majority were commercial travelers and postal clerks; they seem to acquire what might be called a professional dread of accidents, and this is an important factor in the neurosis. The susceptibility to suggestion is extreme and a physician's incautious remark may start or aggravate the neurosis. The jar of the trains does not seem to predispose the trainmen to nervous trouble, as these formed only 12 per cent. of the total. Only 0.465 per thousand developed neuroses among 25,793 employees who met accidents of various kinds in 1909 , and the proportion has been the same since. Ciampolini states that after the question of indemnity had been settled the neurosis gradually subsided with final complete recovery.

\section{INFECTION OF NASAL CAVITY FROM DISEASED TOOTH-ROOT}

\author{
SPECIMEN SHOWING PATHWAY OF INFECTION \\ THROUGH THE MAXILLARY SINUS*
}

\author{
WILLIAM REDFIELD BUTT, M.D. \\ PIILADELPHIA
}

The possibility of the passage of an infection upward from a decayed tooth-root through the maxillary sinus to the nasal cavity is a matter of common

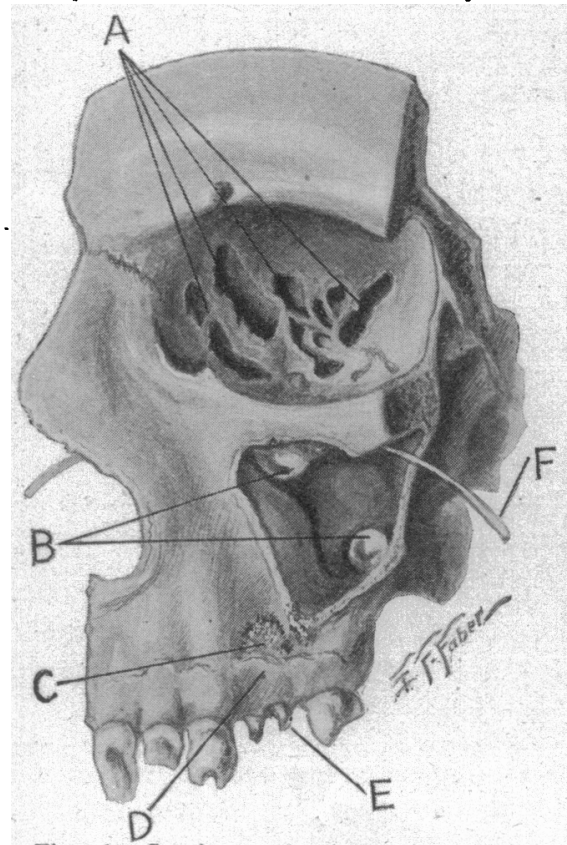
medical knowledge. The. rhinologist frequently finds in this seauence of events a long - delayed explanation, esp_cially of trifacial neuralgias as well as various nose and throat conditions.

The accompanying illustrations are from a post-mortem specimen in which the pathway of such an infection. is $\mathrm{st} \mathrm{rikingly}$ marked out by $\mathrm{p}$ a $\mathrm{th}$ o 1 o g i c changes, namely, a necrotic area Fig. 1.- Specimen showing: $A$, ethmoidal around the root to be free from any evidence of disease; $B$, of a first. (or polypi (upper one located at the lip of the ostium ); $C$, area of necrosis around tooth-root $D$, fistula from tooth-root opening on the gum; $E$, diseased first bicuspid; $F$, bristle passed
from the maxillary sinus into the nose through
the normal ostium.

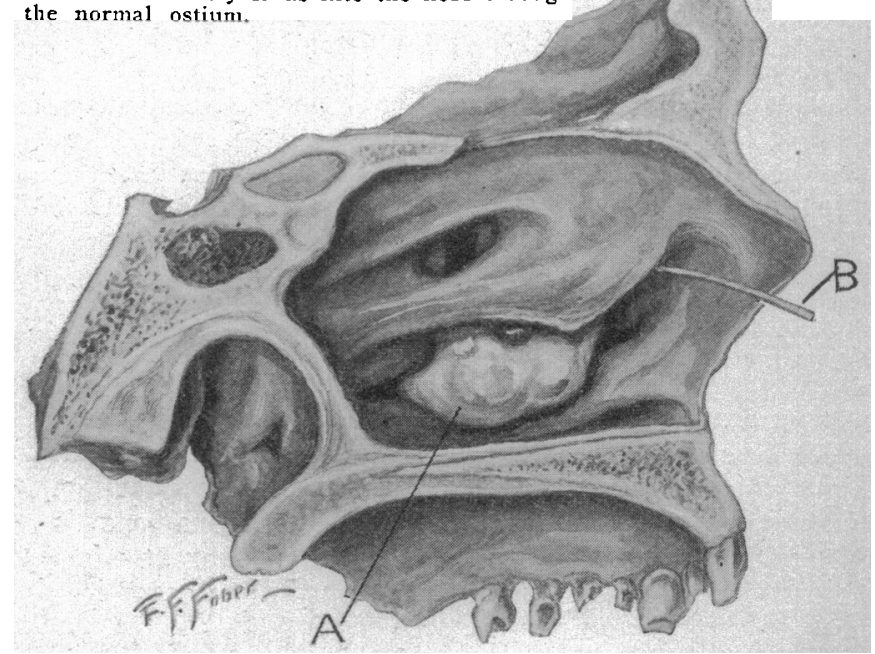

Fig. 2.-Specimen showing: $A$, polyp in middle fossa of the nose growing from the lip of the unciform groove; $B$, bristle passing through the maxillary ostium beneath the middle turbinate.

second) bicuspid tooth, a fistula from this area opening on the gum, changes in the mucous membrane of the maxillary sinus adjoining this area and with polyp formation especially around the ostium, both on the maxillary sinus side and in the nasal cavity.

* Exhibited before the Section on Oto-Laryngology of the Philadelphia College of Physicians, and the Philadelphia Pathological Society. 
The ethmoidal sinuses, opened from the orbital aspect, are seen to be uninvolved.

A point of interest in Figure 2, which, however, has no relation to the foregoing, is this: The sphenoid sinus in this case is represented by a very small depression on the back wall of the nasal cavity (hidden in the illustration) while the space below the sella turcica, usually occupied by the sphenoid sinus, consists of a thick mass of cancellated bone. The cavity shown in this region is not the sf henoid sinus, but was made artificially with the curet in searching for it. It is comforting to the surgeon working in this region to know that when the sphenoidal sinus is absent its territory is usually occupied, as shown in Figure 2, by a thick mass of bone.

1701 Chestnut Street.

\section{CEREBROSPINAL EXAMINATIONS IN "CURED" SYPHILIS}

CASES IN WHICH THE BIOLOGIC METHOD AS A CONTROL HAS BEEN USED *

B. C. CORBUS, M.D.

Instructor in Genito-Urinary Diseases, Rush Medical College CHICAGO

Since the application of the Wasscrmann reaction to the blood-serum as a biologic control in the treatment of syphilis, we have rested securely in the thought that this method of management, if applied correctly, was the last word in the prognosis of this disease. All authorities have recommended it as the most dependable aid which we have in the control of our treated cases of syphilis, and truly it is; but as discovery after discovery has followed in quick succession, each marking an epoch in our knowledge of syphilis, it is but natural that our opinions should change.

Noguchi's ${ }^{1}$ demonstration of the Spirochaeta pallida in the brains of paretics has opened up a new phase of the infection, and whereas we before thought that this localization was only the end-result of the infection, we now know differently. That the spirochetes involve the central nervous system early, in a large percentage of cases, was little dreamed of three years ago, and only within the last year and a half has the management of this class of infections been given serious thought by the average syphilographer.

Since the application of lumbar puncture with spinal fluid examinations, many cases showing a negative blood-serum Wassermann for from eighteen months to two years without treatment show subarachnoid involvement. In the light of our present knowledge, lumbar puncture with spinal fluid examinations is demanded in all cases of syphilis, no matter at what stage of the disease, as a control on future complications of the nervous system.

An apology is offered for referring to the diagnosis of syphilis at the time of the presence of the primary lesion, as every syphilographer knows exactly the value of an early diagnosis, especially before the Wasse:mann is positive; but ever since the arrival of the easy water-soluble neosalvarsan, the treatment of this dis-

* Read before the Section on Dermatology at the Sixty-Fifth Annual Session of the American Medical Association, Atlantic City, N. J., June, 1914.

1. Noguchi, Hideyo: The Transmission of Treponema-Pallidum from the Brains of Pareics to the Rabbit, The Journal A. M. A., July 12,
1913 , p. 85 . ease has passed out of the hands of the specialist into the hands of everybody with a medical degree. It is safe to prophesy that our insane hospitals in the future will contain a larger percentage of cases with irvolvement of the central nervous system than they do to-day. Too little heed is paid to this golden opportunity, and too great a dependency is placed on a few ill-timed "shots" with no consideration whatever of the subarachnoid space.

Since October, 1913, I have performed lumbar puncture with spinal fluid examinations on as. many patients "biologically cured" as I could induce to return for examination. Of these so-called "cured cases," excluding syphilis with cerebrospinal manifestations, I have found spinal fluid involvemont in 18.3 per cent. Many patients resting securely in the faith of a persisting negative Wassermann and good health refused to permit this procedure.

\section{DISCUSSION OF THE TABLE}

The accompanying table shows 6 cases of primary syphilis, 18 of secondary syphilis, 4 of tertiary syphilis, 14 of latent syphilis, 1 of congenital syphilis, and 6 of syphilis with cerebrospinal manifestations; of the last 6 cases, 2 are of incipient tabes, 1 of tabes, 2 of cerebrospinal syphilis and 1 of complete optic atrophy.

The original object of this investigation was to ascertain whether or not the "cure" had been complete and if not, the reason.

Mott $^{2}$ long ago called attention to two factors which play a rôle in the involvement of the nervous system: (a) the specific form of spirochcte, and $(b)$ poor resistance on the part of the host. Having studied these cases with this idea in mind, I have deduced the conclusions which are given below.

\section{PRIMARY SYPHILIS}

All the cases herein recorded wcnt to a satisfactory termination, and $I$ believe are permanently cured. The largest quantity of salvarsan given to a single pa.ient, together with rubbings, was $4.2 \mathrm{gm}$; the smallest was $2.4 \mathrm{gm}$.

\section{SECONDARY SYPHILIS}

In Case 8 the patient, D. H. D., of average nutrition, had been infected nine years, and spirochetes were demonstrated in the muuth lesions before treatment was begun. On account of the length of infection before treatment, the patient being a confirmed alcoholic, subarachnoid involvement might be suspected. Examination was negative.

In Case 9 the patient, H. R. A., aged 38, was strong and robust, and spirochetes were present after five years. Jan. 27, 1914, spinal fluid examination was positive. After intensive intravenous salvarsan, spinal fluid examination April 29, 1914, showed Wassermann negative, cells 3 per cubic millimeter, Nonne negative. The total quantity of salvarsan given was $4.5 \mathrm{gm}$.

Patient 10, G. K. L., aged 51, was very well preserved. There was cerebrospinal involvement at eighteen months. This patient has had fourteen injections of salvarsan intravenously, being a total of $7 \mathrm{gm}$., together with rubbings by a professional, and four injections intraspinally, one after the Swift and Ellis ${ }^{3}$ technic, 20 c.c. of serum being given, and three after the method of Ravaut, ${ }^{4} 4 \mathrm{mg}$. being given at each injection. The spinal fluid is still involved.

Patient 11, C. G., aged 28, strong and vigorous, came under observation during the secondary period. The blood Wassermann always vacillated; spinal fluid examination, Deccmber, 1913, was positive, Nonne positive, and lymphocytes 25 per cubic millimeter. Since severe persistent intra-

2. Mott: In Power and Murphy's System of Syphilis, Oxford Press, 1908, ii, iv.

3. Swift and Ellis: New York Med. Jour., 1912, xcvi, 53. 